\title{
MOVIMIENTOS SOCIALES, GOBIERNOS PROGRESISTAS Y ESTADO EN AMÉRICA LATINA: transiciones, conflictos y mediaciones
}

\section{MOVIMENTOS SOCIAIS, GOVERNOS PROGRESSISTAS E ESTADO NA AMÉRICA LATINA: transições, conflitos e mediações}

\author{
Breno Bringel* \\ Alfredo Falero* *
}

\begin{abstract}
Este artículo busca analizar la América Latina contemporánea en clave procesual y relacional, a partir de la relación entre movimientos sociales, gobiernos progresistas y Estado. Su objetivo es comprender el ciclo político progresista que se abre en el cambio de siglo de forma paralela a cambios políticos, sociales y económicos más estructuradores. De forma más específica, el texto discute y examina: i) la necesidad de captación del proceso social y la diversidad de formatos de interacción entre movimientos sociales, gobiernos progresistas y Estado en la región; ii) la especificidad de la región, su posición dependiente en el mundo y sus consecuencias sociales y políticas, incluyendo la transformación de la forma Estado, cada vez más transnacionalizado; iii) los principales ejes de conflicto social, oriundos en buena medida de las mediaciones sociopolíticas y de las contradicciones político-económicas; iv) los tipos de gobiernos, los límites del progresismo y las tensiones entre gestión estatal y movimientos sociales.

Palabras-Clave: Movimientos sociales. Gobiernos progresistas. Estado. América Latina.
\end{abstract}

\section{INTRODUCCIÓN ${ }^{1}$}

Bolivia, octubre de 2011. Tras muchas protestas, el gobierno de Evo Morales firma la Ley Corta 180, que declara intangible el Territorio Indígena y Parque Nacional Isiboro-Secure (TIPNIS). Más allá de un caso aislado, el caso TIPNIS, polémico por la propuesta de construcción de una carretera que pasaría por este territorio, supuso una importante inflexión en las relaciones de convergencias entre los

\footnotetext{
* Universidade do Estado do Rio de Janeiro (UERJ). Instituto de Estudos Sociais e Políticos (IESP).

Rua da Matriz, 82, Botafogo. Cep: 22260-100. Rio de Janeiro - Rio de Janeiro - Brasil. brenobringel@iesp.uerj.br

${ }^{* *}$ Universidad de la República (UDELAR).

UDELAR, Facultad de Ciencias Sociales, Constituyente, 1502, $5^{\circ}$ piso. Montevideo - Uruguay. alfredofalero@gmail.com

${ }^{1}$ Una primera versión de este texto fue elaborada durante el segundo semestre de 2014 como insumo para el curso "América Latina: Movimentos Sociais e Tranformações Regionais" por nosotros impartido en el Programa de Posgrado en Sociología del IESP-UERJ y luego presentado en el $38^{\circ}$ Encuentro Anual de ANPOCS, 27 a 31 de octubre de 2014, Caxambu (Brasil). Dicha versión inicial se publicó con circulación restringida, como Cuaderno de Trabajo del Núcleo de Estudios de Teoría Social y América Latina (NETSAL). En este proceso, hemos recibido diversos comentarios que nos llevaron a actualizar y reformular buena parte del texto, hasta llegar a esta versión que se publica en el Caderno CRH. Agradecemos los comentarios recibidos en estos espacios, bien como las sugerencias de Enara Echart y Denise Vitale a esta versión final.
}

movimientos sociales y el gobierno boliviano. El caso no se cerró de forma definitiva, pero reveló las contradicciones profundas entre la propuesta de refundación del Estado centrada en el campo popular-plurinacional, con una reorganización de la relación entre sociedad y naturaleza, y la pervivencia de prácticas desarrollistas y extractivistas.

Brasil, junio de 2013. Movilizaciones masivas y heterogéneas se extienden por todo el país y desatan una profunda crisis política que tambalea el Partido de los Trabajadores (PT) en, al menos, un doble sentido: por un lado, como gobierno, en su capacidad de construcción política y de generación de gobernabilidad; y, por otro lado, como partido, en su papel histórico de mediador del campo popular-democrático brasileño, forjado en el contexto de luchas contra la dictadura. Si lo primero evidencia un deterioro en términos de legitimidad política, lo segundo abre una brecha todavía más profunda en el tejido social, dada la desconexión con los actores sociales emergentes que desafían tanto el PT como los demás sujetos que emergieron en el ciclo de la redemocratización política de los 1970/1980. 
Ecuador, agosto de 2015. Marchas y paros nacionales convocados por los movimientos sindicales e indígenas contra el gobierno Correa acaban con centenas de heridos y detenidos en varias partes del país, incluyendo una periodista-académica brasileña y conocidos líderes de la Confederación de Nacionalidades Indígenas del Ecuador (Conaie) y de la Confederación Kichwa del Ecuador (Ecuarunari). Ante la ausencia de confluencias con los movimientos populares, como ocurrió en el proceso boliviano, episodios como este llevan a una ruptura todavía mayor y a una agudización del conflicto, existente desde hace varios años, entre los movimientos indígenas y el gobierno ecuatoriano.

¿Qué pueden decir estos eventos de protesta sobre la relación entre movimientos sociales, gobiernos progresistas y Estado en América Latina? Interpretados de forma casuística y singular, muy poco. Sin embargo, si adoptamos una mirada histórica, comparativa y global, se pueden esbozar algunas claves de análisis para captar las relaciones complejas entre estos actores en coyunturas críticas. Cuando la relación de los movimientos sociales se establece con gobiernos con los cuales éstos tienen algún tipo de afinidad (histórica, político-ideológica, organizativa o programática), en tiempos de transformación profunda del papel del Estado y del capitalismo, nuestras lentes analíticas deben ser revisadas. un cambio de época, vivimos, quince años después, una crisis de los gobiernos con sensibilidades de cambio en casi toda la región. De hecho, un conjunto de evidencias sugieren que presenciamos un tránsito de las tensiones iे creativas (fruto de una serie de retroalimentaciones y sinergias generadas entre movimientos, partidos y gobiernos durante el cambio de siglo y la primera década del siglo XXI) a las tensiones paralizantes (caracterizadas por posiciones más polarizadas y menos dialógicas tanto de movimientos como de gobiernos que pierden progresivamente su base social y, en la mayoría de los casos - aunque no como consecuencia causal -, también su base electoral).

La hipótesis del fin del ciclo progresista latinoamericano, sin embargo, debe ser leída con mucha cautela. No se puede establecer un juicio taxativo sobre el fin del proceso socio -histórico caracterizado por la confluencia de fuerzas de las izquierdas sociales y políticas, bien como de presencia de gobiernos progresistas y nacional-populares (entre otros rótulos utilizados, menos afortunados, como socialdemócratas y populistas), sin cruzar diversas variables y escenarios posibles.

Tres factores principales exigen prudencia. En primer lugar, el ciclo progresista no implicó, en un sentido estricto, a la totalidad de la región, aunque haya influido en general en toda ésta. De este modo, la zona del Pacífico fue menos permeable a esa mutación, mientras lo ocurrido en Paraguay fue particularmente débil y temporalmente breve. Ya México, junto a otros países, aparece desde hace años como más atado a las lógicas de dominio de Estados Unidos y a las dinámicas propias de descomposición social vinculadas a la narcopolítica que bloquean cualquier rotación política creíble de gobiernos.

En segundo lugar, aunque parte de la literatura especializada (Castañeda, 2006; Pereira da Silva, 2011) haya intentado explicar la emergencia de los gobiernos progresistas en la última década y sus diferencias, más allá de dicotomías insuficientes - como reformistas y radicales, neoliberales y postneoliberales -, todavía no se ha explorado adecuadamente la cuestión de cuán progresista fue el dichoso "giro a la izquierda" y cómo, en muchos casos, se basó en patrones de acción ambivalentes y contradictorios.

En tercer lugar, la apertura societaria y política vivida hoy en los diferentes países de la región puede tener desenlaces imprevisibles y pasibles de oscilaciones según las especificidades de las trayectorias, dinámicas y procesos locales y nacionales. Esto es fruto, a su vez, de la existencia de patrones particulares 
de relaciones establecidas entre el Estado y la sociedad, bien como de la existencia de tipos diversos de conflictos y movimientos sociales.

Pese a estos matices, que contemplan la diversidad y la contingencia, asumimos un doble movimiento teórico-metodológico: en términos socio-espaciales, entendemos América Latina como unidad, dentro de su diversidad de situaciones, experiencias y posiciones (Falero, 2012a); en términos temporales, buscamos captar la historicidad de las luchas sociales y conflictos que se despliegan a lo largo de las últimas décadas en su relación con los gobiernos y Estados. De este modo, no podemos disociar el ciclo de gobiernos progresistas de los ciclos de protestas de los movimientos sociales, sean aquellos que contribuyen a elegir nuevos gobiernos o aquellos que ayudan a destituirlos.

El inicio y el cierre de ciclos políticos (nunca totalmente fijos y estrictamente delimitados) están siempre asociados a coordinadas y cambios políticos, sociales y económicos estructuradores. Este artículo privilegia los cambios en el terreno económico (del capitalismo y de la inserción de América Latina en el mundo), político (las transformaciones de la forma Estado) y social (las oscilaciones en los ejes de conflicto social), para proponer formas de entendimiento de los tipos de gobiernos, los límites del progresismo y las tensiones entre gestión estatal y movimientos sociales. En otras palabras, en vez de centrarnos exclusivamente en el análisis de la actuación de los gobiernos en sí, se enfatizan sus contextos de acción y de relación.

El objetivo principal es contribuir a la apertura de nuevas posibilidades de análisis de las relaciones entre movimientos sociales, gobiernos progresistas y Estados. Lejos de una contribución definitiva, este ejercicio debe ser entendido como una provocación al debate y un esfuerzo provisional dependiendo del cierre del proceso socio-histórico considerado.

\section{SOBRE NUESTRA CAPACIDAD DE CAPTACIÓN DEL PROCESO}

El ciclo de victorias electorales progresistas que se inicia con la elección de Hugo Chávez en 1998 no se puede entender sin el ciclo regional de movilizaciones que lo acompañó. Éste se inicia a principios de la década de 1990, con un incremento de la articulación de las luchas regionales, la resistencia directa a la propuesta del Área de Libre Comercio de las Américas (ALCA) y la generación de campañas puntuales y espacios permanentes de convergencia. Acompañado de conflictos más localizados (aunque no aislados o localistas) en los territorios nacionales, se apostó, no sin tensiones, por la integración regional y por la creación de plataformas y redes de convergencia entre movimientos, colectividades más descentradas, campañas, partidos y sindicatos de izquierda (Bringel; Cabezas, 2014). En la segunda mitad de la década de 1990 ya son visibles numerosas expresiones de lucha como se dará cuenta más adelante mediante una tipología.

Esto fue crucial para que fuerzas de izquierda llegaran al gobierno. En algunos casos se generaron formaciones sociopolíticas de nuevo cuño (Bolivia, Ecuador, Paraguay y Venezuela), que emergieron con este ciclo de movilizaciones y disputaron elecciones, mientras, en otros, primaron formaciones más tradicionales (Argentina, Brasil, Chile, El Salvador, Nicaragua y Uruguay), forjadas en décadas anteriores, principalmente en las luchas contra la dictadura. El frescor de las nuevas fuerzas y la experiencia de aquellas más consolidadas son elementos centrales no sólo para cuestionar los rumbos tomados por los gobiernos, sino también para analizar las diferentes relaciones con los movimientos sociales.

Estas relaciones se complejizaron de forma creciente cuando pasaron a gobernar los compañeros. ${ }^{2}$ Ello requirió creatividad e inno-

${ }^{2}$ Este es el título de un documental audiovisual producido por el Núcleo Interdisciplinario "Pensamiento Crítico en América Latina y Sujetos Colectivos" de la Universidad de la República de Uruguay que incluye entrevistas con mili- 
vación en los trabajos académicos, que han enfatizado la necesidad de ir más allá de interpretaciones dicotómicas (tales como autonomía versus institucionalización, basismo versus cooptación, conflicto versus cooperación, reforma versus revolución, etc.) que, en buena medida, han marcado las interpretaciones clásicas sobre las relaciones entre los movimientos sociales y el Estado en la región.

Entre las alternativas propuestas en los últimos años por la literatura especializada brasileña (muy marcadas, hay que decirlo, por la experiencia de las legislaturas del PT en el gobierno nacional), sobresalen aquellas que enfatizan la mayor permeabilidad estatal, la heterogeneidad del Estado y sus formas de interacción, a partir de miradas sobre los repertorios de interacción (Abers; Serafim; Tatagiba, 2014), las interacciones socioestatales (Nascimento, 2014), las imbricaciones Estadosociedad (Losekann, 2014) las "redes sociales y de movimientos" (Scherer-Warren, 2013), los "mediadores" (Lavalle; Bullow, 2015), el tránsito institucional, la trayectoria de militantes y las fronteras de los movimientos, la sociedad civil y del Estado (Silva, 2015).

En otros países de América Latina, la preocupación por entender proyectos de refundación del Estado en la región andina (Santos, 2010) y la creación de nuevos instrumentos políticos o partidos-movimiento en Paraguay, Bolivia, Ecuador y Venezuela ha llevado a que varios autores subrayen las nuevas relaciones 害 entre movimientos sociales y los sistemas po¿̀ líticos (Mirza, 2006) y la importancia de conके siderar no sólo aspectos coyunturales de la re될 lación Estado-movimientos, sino también las \& matrices sociopolíticas (Garretón, 2001) y las iิ matrices político-ideológicas (Svampa, 2010;

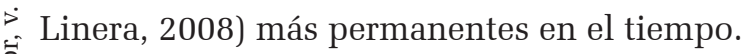

el Estado casi siempre sólo en su dimensión de las relaciones internas y nacionales. Aunque las redes transnacionales (Bringel; Falero, 2008; Bulow, 2010), la internacionalización de los movimientos (Bringel, 2015; Pleyers, 2012; Tarrow, 2005) y los movimientos globales (Bringel; Domingues, 2015; Della Porta, 2007) sean muy estudiados en el marco del activismo contemporáneo, menos énfasis es dado a cómo: a) el Estado se ha reconfigurado profundamente en el siglo XXI a través de una confluencia de dinámicas, fuerzas y actores diversos (sociales, políticos y, principalmente, económicos); b) cuáles son las implicaciones de esto en su relación con los movimientos sociales y en la capacidad de actuación de los gobiernos.

El segundo problema se puede plantear en los siguientes términos: la sociología de los movimientos, al leer la relación entre los movimientos sociales y el Estado, teniendo como objeto principal los movimientos, obvia, muchas veces, el otro lado de la moneda: el Estado y su complejidad, su capacidad de agencia, su historicidad, sus disputas internas, sus correlaciones de fuerzas y su lógica selectiva en la selección y en la predisposición al diálogo con sus interlocutores.

Estas consideraciones nos llevan a pensar que la relación entre el Estado y los movimientos sociales debe ser entendida en el marco de un campo relacional amplio, donde intervienen varios tipos de acciones, actores sociales y políticos y dinámicas de acumulación del capital. La idea de lo relacional remite a una vasta bibliografía dentro de la cual cabe subrayar, en una mirada más general, la postura de Bourdieu en cuanto a pensar relacionalmente (Bourdieu; Wacquant, 2014), pero también la idea de proceso social y los usos de la teoría cuando se visualiza específicamente América Latina (Falero, 2015). Si nos adentramos en los estudios contemporáneos sobre conflicto político y movimientos sociales, cabe subrayar que el "giro relacional" es una tendencia bastante fuerte cuando se postula den- 
tro de las agendas hegemónicas que el estudio de estos actores sociales no puede quedar circunscrito a las organizaciones sociales y (o) a los movimientos sociales entendidos como unidades analíticas autosuficientes (McAdam; Tarrow; Tilly, 2001). A pesar de ello, los autores vinculados al proyecto contentious politics se centran principalmente en las interacciones públicas, episódicas y colectivas entre demandantes y demandados, con énfasis en los gobiernos y en las instituciones, prácticamente dejando de lado los actores, las prácticas y los escenarios económicos.

En esta línea, es fundamental pensar los movimientos más allá de los movimientos, en relación con actores diversos (Estados, empresas, grupos sociales específicos); fenómenos afines, pero diferenciados (nacionalismos o migraciones); e impactos múltiples (políticos, pero también societarios, económicos y culturales). En definitiva, captar los eventos de protesta dentro de una mirada procesual que contemple las tendencias socio-históricas, las mediaciones sociopolíticas y las dinámicas estructuradoras de sociedades que se ubican en la periferia capitalista.

El resultado de las relaciones entre estos actores y dinámicas es producto de diferentes articulaciones posibles, en función de un conjunto de variaciones de los niveles señalados. Si la clave está en la caracterización de mediaciones, discutiremos, a partir de ahora, cuatro elementos centrales: a) las variaciones posibles dentro de la inserción de América Latina en la economía-mundo; b) las transformaciones de la forma Estado en función de procesos globales, regionales y nacionales; c) la actividad del conjunto de movimientos y organizaciones sociales y su capacidad de transformar el Estado y la gestión de gobierno, bien como de influir en el posicionamiento que adopten los partidos políticos; d) las dinámicas de gobiernos cuya dinámica de juego se ve restringida en función del punto a) e interpelada en abrir otros horizontes de posibilidades en función del punto c).

\section{LA UBICACIÓN DE AMÉRICA LATI- NA EN EL MUNDO}

A pesar de la emergencia de gobiernos progresistas y de una posición geopolítica relativamente más autónoma de la región en el mundo (Cairo, 2008), todavía sigue firme, en el marco de la actual transición sistémica global, la posición de América Latina como proveedora de materias primas a la economía-mundo. Si bien se logró pasar página en la década de 1990 al Consenso de Washington, el cambio de siglo marca la irrupción del Consenso de las Commodities (Svampa, 2013), definido por el incremento de los precios internacionales de las materias primas y los bienes de consumo, cada vez más demandados por las potencias centrales y emergentes.

Tampoco hay que desdeñar la composición sectorial de la inversión extranjera directa (IED) que, según el informe de 2012 de la Comisión Económica para América Latina y Caribe (CEPAL), sigue teniendo un peso considerable. Incluso en Brasil, crece el peso económico de los servicios (una categoría en verdad muy poco explicativa pues puede integrar elementos muy diversos) y de las actividades basadas en recursos naturales a expensas de una menor participación de la industria manufacturera.

Este escenario tiene efectos en la composición y en las características de la fuerza de trabajo y, por tanto, en la configuración de los agentes participantes de las luchas sociales, en la importancia que tienen los movimientos sociales relacionados al territorio, en los conflictos que se generan a partir de la forma en que se expresa su implantación - la más compleja es la forma enclave actualizada (Falero, 2012c) - y en la infraestructura necesaria para la exportación. Dentro de este cuadro general, hay casos diversos. Por ejemplo, Uruguay tiene una población de base rural del 5\%, algo muy diferente a lo que ocurre en la región andina o centroamericana. Es decir, en ese caso, el movimiento que integra 
la temática ambiental (luchas medioambientales vinculados al uso de transgénicos, la forestación o el posible desarrollo de actividades mineras, entre otras) solo puede tener proyección manejando ese condicionamiento urbano en la expresión de las demandas.

Asimismo, el efecto China y la caída del precio de las materias primas tienen efectos directos en el excedente que los Estados tienen para canalizar demandas de movimientos sociales y procurar matizar las consecuencias sociales de la extrema desigualdad en la región. En otras palabras, el elemento geoeconómico, no siempre analizado con propiedad en el estudio de las relaciones entre movimientos sociales y Estado, desnuda las debilidades de las posibilidades de cambio y refuerza las contradicciones del progresismo, sobretodo de los gobiernos que han apostado por la vía (neo) desarrollista.

Viejas tendencias (entre ellas, aquella bautizada, en el debate regional, como la maldición de los recursos naturales) se enmarcan en un nuevo escenario geopolítico y geoeconómico. Mientras buena parte de la franja del Pacífico (particularmente, Chile, Perú, Colombia y México) mantiene, en términos generales, su alineamiento con Estados Unidos - aunque sin desconectarse de la región en su conjunto -, la región que proyecta más incertidumbre es la del Atlántico, con el epicentro en Brasil. Las superestructuras políticas integradoras (prin๑ cipalmente Mercado Común del Sur - MERì COSUR, Unión de Naciones Sudamericanas 新 - UNASUR y Comunidad de Estados Latinoa. mericanos y Caribeños - CELAC) muestran, a के su modo, las complejidades de una transición 될 sistémica con pérdida relativa de peso de Es\& tados Unidos, y los nuevos bloques regionales 今 - todavía superpuestos y en disputa - parecen $\vec{t}$ tener una capacidad bastante limitada para revertir la dependencia económica y fomentar, de manera más significativa, la integración en el plano cultural y político.

En este marco de tensiones geopolíticas y geoeconómicas, corresponde recordar una tendencia intrínseca al capitalismo como sistema global: la polarización entre regiones centrales de acumulación y regiones periféricas (Amin, 2003; Falero, 2012b). Esta conocida tesis, ampliamente desarrollada por los teóricos de la dependencia, ni sugiere la existencia de posiciones de poder eternamente fijas, ni tampoco caduca por la crisis de 2008, o porque se esté en una fase de conexión global sin precedentes de la mano del mayor peso de la comunicación, la información (en un sentido amplio) y el conocimiento en la valorización del capital.

A pesar de propuestas como la del gobierno ecuatoriano de generar un cambio de matriz productiva centrada en la economía social del conocimiento, en los centros de acumulación se investigan y se generan los nuevos procesos científico-tecnológicos y se conserva el know how de múltiples actividades intensivas en conocimiento, mientras algunas actividades industriales se desplazan a la periferia para disminuir los costes. En otras palabras, la polaridad centro-periferia no desaparece, sino que se exacerba sobre nuevos parámetros, tales como los derechos de propiedad intelectual, las patentes y la extracción de biodiversidad.

No se puede obviar, en este sentido, que las grandes decisiones globales no se toman en la periferia capitalista. Sigue existiendo una transferencia de excedentes desde ésta a los centros de acumulación y el discurso del desarrollo (que observa carreras lineales y cíclicas de países o carreras nacionales, vinculado todavía de manera hegemónica al crecimiento económico) ha sido revitalizado más como un eficaz mecanismo de contención geopolítica de lo social que como una guía o rumbo de la trayectoria hacia donde efectivamente se va.

Esta actualización de la polarización centro-periferia, bien como el nuevo escenario geopolítico global aludido, afecta la capacidad de los Estados latinoamericanos de tomar decisiones relevantes y se sustenta en la reproducción de redes de poder global más o menos conocidas: grupos tecnocráticos y burocráticos con intereses específicos dentro de los Estados- 
nación, grupos económicos locales conectados globalmente, empresas transnacionales, agencias multilaterales, entre otros. En esta forma de despliegue espacial del poder, se desnacionalizan los Estados y se reproducen las articulaciones (social y geográficamente asimétricas) que cristalizan en esa polaridad global. Pensar las relaciones entre movimientos sociales y Estado en el siglo XXI es, en consecuencia, considerar un modo de funcionamiento social con fronteras mucho más porosas.

Considerando los gobiernos usualmente clasificados como progresistas, se puede observar, por un lado, como lo ha hecho Campello (2016), la limitación impuesta por los mercados globales. Por otro, también se puede constatar que las bases de poder de esa desigualdad, en algunos casos, se intentó redefinir mínimamente (Argentina, Bolivia, Ecuador y Venezuela) y, en otros, ni siquiera se intentó (Brasil, Chile, Uruguay), pero, en todos los casos, la cuestión central pasa a ser la ponderación de la conexión entre la proyección posible de un orden progresista en una economía-mundo más adversa para sus exportaciones.

\section{MOVIMIENTOS SOCIALES Y TRANSFORMACIONES DE LA FOR- MA ESTADO}

Unida a las transformaciones geoeconómicas globales y regionales, la forma Estado tiende a cambiar, y es fundamental reintroducir este anclaje espacio-temporal del Estado en el análisis de los movimientos sociales. En muchos análisis actuales sobre la relación entre los movimientos sociales y el Estado, éste aparece como algo dado, prácticamente naturalizado. Quizás eso se deba a que buena parte del esfuerzo analítico se vuelca al entendimiento de los movimientos sociales en sí (con el consecuente desarrollo teórico del debate sobre conflicto, acción colectiva, identidades, repertorios, etc.), relegando a un segundo plano el debate teórico sobre el Estado. Sin embargo, a pesar de sus asimetrías y diferencias, un análisis relacional de estos actores debiera dar, en términos analíticos, un peso relativamente análogo a cada uno de ellos, de forma que podamos entender con mayor precisión sus recorridos históricos, sus formas de acción, su heterogeneidad interna, las diferentes correlaciones de fuerzas a corto plazo y matrices sociopolíticas más permanentes en el medio y largo plazo.

Lecturas institucionalistas y formalistas del Estado, en buena medida racionalistas y universalistas, tienden a reificarlo, reproduciendo los límites analíticos de las concepciones contractualistas y organicistas que fundaron la teorización sobre el Estado. No se trata, obviamente, de idolatrar o demonizar al Estado, de depreciar la importancia de las instituciones, los funcionarios y la burocracia, ni tampoco de entender el Estado como mero espejo de la sociedad (algo muy en boga hoy, cuando se habla de una deriva conservadora), sino de dotar nuestro aparato analítico sobre la relación entre movimientos sociales y Estado de mayor historicidad, espacialidad y contingencia. La construcción de una teoría crítica del Estado conectada a las teorías de los movimientos sociales constituye, de este modo, un desafío fundamental que permitiría, además, reconectar el debate fundante de los movimientos sociales en la modernidad con las transformaciones de quien, todavía hoy, es su actor político hegemónico.

El formato emancipatorio de los movimientos tuvo, en general, en el Estado-nación, un objetivo central de llegada en tanto herramienta para cambios descolonizadores, anti-imperialistas, constructores de derechos o directamente visualizándolo como núcleo para el desarrollo de una sociedad no capitalista (Bringel; Domingues, 2012). En el seno de varias luchas sociales transformadoras durante buena parte del siglo XIX y XX, el Estado fue visto como expresión de dominación de clase y como organización sociopolítica transitoria (caso de Marx y del marxismo en general). 
También se vio como agente autoritario, jerárquico y de control social para muchas luchas autónomas y libertarias (como se puede comprobar tanto en el anarquismo, como en matrices comunitaristas e indigenistas).

Sea como espacio a conquistar para su posterior disolución y construcción del socialismo o del comunismo, sea como enemigo frontal, el Estado se ha visto, en las teorizaciones abarcadoras más consistentes sobre el tema, como proceso y como relación social y objetiva de poder (Gramsci, 1949; Poulantzas, 1968; Foucault, 1976; Mann, 1984). Una de las mayores virtudes de estas teorizaciones ha sido el análisis de correlaciones de fuerzas y procesos específicos anclados en el espacio (teniendo en cuenta la particularidad de los lugares y las implicaciones de la territorialidad del Estado asociada a su soberanía) y en el tiempo (analizando la transformaciones del Estado en diferentes momentos históricos).

El Estado, en América Latina, como constructo histórico en la periferia mundial, ha tenido un proceso de formación particular (Borba, 2014; Centeno, 2002; López-Alves, 2003) y tiene un desarrollo contemporáneo convergente con esta matriz espacio-temporal y con los procesos sociopolíticos, culturales y geoeconómicos que atraviesan la región. A partir de la década de 1970, momento para el cual Guillermo O’Donnell desarrolló una consistente interpretación - de lo que denominó como Estado burocrático ล. -autoritario (O’Donnell, 1982), el capitalismo se volvió progresivamente cada vez más global. solo es posible aludir brevemente algunas de las razones globales del debilitamiento en relación al proceso de transnacionalización: a) el creciente peso de las empresas transnacionales y su capacidad para generar cursos de acción de los gobiernos o para lograr excepcionalidades en el territorio de los Estado-nación; b) el incremento del poder de los organismos multilaterales y de determinadas agencias globales (como las calificadoras de riesgo) para (re)direccionar la política económica y para incidir en la gestión pública; c) la firma de tratados bilaterales asimétricos de protección de inversiones, que limitan cursos de acción de los gobiernos, principalmente aquellos de regiones periféricas que suscriben acuerdos con los países y regiones centrales de acumulación global; d) la creación de bloques regionales y generación de dinámicas comerciales, productivas, políticas y sociales inevitablemente supranacionales; e) los problemas cuyos efectos exceden las posibilidades de decisión de un Estado-nación (cambio climático, políticas derivadas de desarrollos tecnológicos, etc.).

Estas dinámicas y tendencias se expresan dentro de los aparatos de Estado y los transforman considerando los parámetros previos. Esto supone un desafío para los movimientos sociales en varios sentidos. Por un lado, las lógicas de funcionamiento político tienden a adaptarse a estas nuevas agendas a costa de presiones y demandas sociales. Por otro lado, las nuevas agendas globales y las dinámicas de extracción de excedentes implican la incorporación de nuevos temas y la generación de propuestas alternativas y de demandas más aglutinadoras capaces de captar la inteligibilidad de diferentes luchas localizadas o territorializadas dentro de un marco más totalizante, como ocurre en el caso de las ideas de soberanía alimentaria y de agroecología frente a la lógica del agronegocio.

En segundo lugar, cambian las lógicas de conflicto y de identificación práctica y subjetiva entre lo que serían los amigos y los enemigos políticos. Por un lado, actores como las empresas transnacionales y nuevos símbolos 
del capitalismo global emergen como enemigos centrales, muchas veces asociadas a los propios Estados, bien por su complicidad implícita o por sus acuerdos explícitos con estos agentes. Por otro lado, actores progresistas, como sindicatos y partidos políticos, cuando victoriosos en contiendas electorales, se mueven progresivamente en la frontera entre amigo y enemigo, hacia un campo de enfrentamiento débil debido a su posición coyuntural, incluso cuando se generan, en el interior del Estado, fuertes tensiones entre el partido en el gobierno y las bases de este mismo partido o alrededor de coaliciones volátiles. Dentro de este escenario, puede ocurrir que determinados partidos estén en el gobierno, pero no logren gobernar.

En tercer lugar, es necesario reconocer una transformación de tipo organizacional del capitalismo, con dinámicas de acumulación flexible, que impacta en la propia gestión, es decir, en la introducción de formas de organización empresarial y en un nuevo papel asumido por las elites empresariales como agentes sociales con capacidad de incidir directamente en cursos de acción nacional y transnacional (Flores, 2006). A efectos de recorte del tem , nos gustaría subrayar un elemento específico de la reconfiguración de la forma Estado en la actualidad, asociado a las dinámicas transnacionales contemporáneas: la emergencia de un concepto de gestión empresarial que atraviesa agentes sociales diversos así como, en general, una perspectiva instrumental, pragmática, muchas veces autodefinida como despolitizada y desideologizada de la gestión.

Para entender estas transformaciones, no hay que caer, sin embargo, ni en el discurso globalófilo del fin del Estado y consecuentes narrativas problemáticas en la periferia capitalista (como la de los Estados fallidos), ni tampoco en aquellas visiones que colocan al Estado como mero apéndice del capital. Hay, de hecho, una dificultad muy grande, en términos teórico-metodológicos, de captar la nueva forma Estado y su asociación con las elites empresariales, las redes tecnócratas y los espe- cialistas locales y globales que revisten de un carácter técnico varias decisiones políticas y (o) proyectos específicos de sociedad. La consecuencia más grave de esto es que el Estado deja de ser considerado en términos de una compleja relación social (expresión, a la vez, de relaciones sociales capitalistas), ya que el mensaje hegemónico transmitido es que se trata puramente de quien y de qué modo se puede administrar o gestionar mejor lo público o lo privado y sus interrelaciones.

Bajo esta lógica, y ante el desdibujamiento de una burguesía nacional fuerte, el proyecto sociopolítico es, no pocas veces, subordinado a lo fiscal, ya que pareciera que no existen intereses particulares e intereses colectivos, pues prácticamente todo desaparece en la figura del gestor. La identificación de la gestión del Estado con el new management de la empresa privada, y con expresiones asociadas como la eficacia, la eficiencia y su carácter emprendedor (Boltanski; Chiapello, 2002) llega a diversos sectores, incluso la propia educación y la universidad.

Finalmente, en cuarto lugar, puede identificarse una transformación de lo que podemos llamar mapas cognitivos que, para esbozarlo en forma muy rápida, se sustenta en un contexto de exacerbada concentración de los medios masivos de comunicación y de profusión de imágenes que borran las fronteras entre lo falso y lo verdadero, la ficción y la realidad, lo intrascendente y lo sustantivo. Se aprecia la emergencia de nuevas gramáticas culturales, proliferación de las redes digitales, profunda mercantilización de la vida y crisis de las agencias de socialización alternativas que fueron propias del siglo XX, como los partidos políticos y los sindicatos.

Si bien no es posible aquí más que aludir al tema, debe ponderarse suficientemente, como desafío para los movimientos sociales en la región, que el plano simbólico (imágenes, palabras, símbolos) adquiere mayor importancia que antes y se vuelve un espacio clave de lucha donde deben disputar formas de ver y pensar el mundo en un terreno de mayor complejidad. 
Por otro lado, la crisis de los actores clásicos de mediación entre sociedad y política, durante el siglo XX, en América Latina, lleva a la emergencia de nuevos formatos de relación entre movimientos y Estado, pero también, muchas veces, a una mayor desconexión entre los movimientos sociales y el resto de la sociedad, principalmente cuando muchos de ellos dejan de lado el trabajo de base para volcarse principalmente en la incidencia política en las instancias institucionales. Habría que preguntarse, de este modo, hasta qué punto el mayor foco de los movimientos, partidos y otros grupos en la presión política hacia los Estados supuso un retroceso en su acúmulo de fuerzas locales y en la constitución y reproducción de sus bases sociales, o si, por otro lado, se trató de una estrategia articulada en planos diferenciados de acción política.

\section{EJES DE CONFLICTO Y LUCHAS SOCIALES EN LA AMERICA LATINA CONTEMPORÁNEA}

Las transformaciones geoeconómicas y de la forma Estado analizadas se asocian a una doble dimensión de la permeabilidad del Estado en la América Latina contemporánea. Por un lado, una mayor permeabilidad interna, que se expresa en la emergencia de gobier๑ nos progresistas y en la complejización de sus N formas de relación e interacción con los movimientos sociales. Por otro lado, una mayor ลे $\sim$ permeabilidad externa, asociada a las fuerzas

\& y procesos políticos que desbordan la territo될 rialidad y la soberanía del Estado-nación, ge$\dot{a}$ nerando tensiones y conflictos típicos de un of Estado transnacionalizado. sociales como objetos delimitados de estudio, lo cual implicaría no captar la variedad de expresividades de los mismos, proponemos visualizar transversalmente las diversas luchas sociales que se fueron conformando en la región en las últimas décadas. Los movimientos sociales se convierten, de este modo, en un recurso heurístico privilegiado para entender elementos que cruzan la realidad latinoamericana e inciden en el campo sociopolítico. En dicha lógica, es clave entender lo que ocurre antes y después de la aparición de gobiernos con diversos tintes de izquierda, dentro de un mismo proceso social en el que se van construyendo demandas y abriendo agendas, pero a la vez generando nuevas tensiones.

Sobre ese criterio y asumiendo el carácter dinámico de estas luchas, puede esbozarse el siguiente esquema general de ejes de conflicto y tipos de luchas sociales, representativas de las tendencias centrales de la conflictividad social en los países y subregiones de América Latina y el Caribe. Se asumen como variables principales los ejes de conflicto, los sujetos sociales y las transformaciones sociales y regionales derivadas de estas contiendas:

Luchas vinculadas al trabajo - Son provenientes de sindicatos o de movimientos sindicales que tienen el formato básico heredado del siglo XX, aunque muchas veces renovadas en nuevos formatos. Conviven, en este campo, luchas salariales y de derechos laborales, por empleo y por la protección social, con nuevas formas de lucha vinculadas a empresas recuperadas, reivindicaciones sobre el trabajo rural en base a cooperativas, entre otras. Se incluye también aquí las luchas del precariado y aquellas vinculadas al no trabajo, es decir, lo que dio lugar a los movimientos de desocupados (como el movimiento piquetero en Argentina) en varios países de la región en el marco de una profunda reestructuración (regional y global) del conflicto laboral.

Luchas vinculadas a la transformación de la forma Estado - Son luchas que van más allá de lógicas electorales y de reformas políticas puntuales, aunque pueden relacionarse con el poder constituyente. Es el caso de plebiscitos y referéndums en Uruguay en el pasado (aunque este también es un recurso utilizado por la derecha política vinculado, por ejemplo, a la seguridad pública) y fueron 
particularmente los casos de Bolivia y Ecuador con los movimientos indígenas, que tienen como bandera central la descolonización y la transformación estructural de la forma Estado hegemónica en la modernidad. Estas luchas suelen estar acompañadas de un ciclo rebelde que, a su vez, desemboca en dinámicas instituyentes propulsoras de nuevas constituciones políticas.

Luchas vinculadas al territorio y a los recursos naturales - Absolutamente centrales en la América Latina contemporánea, son luchas de base territorial vinculadas a conflictos que abarcan ejes como la biodiversidad, la minería, el (neo) extractivismo, el agua o la construcción de infraestructura. Son luchas que, en su defensa de los bienes comunes, disputan los sentidos y los modelos de desarrollo, con una amplia gama de actores involucrados, con destaque para intereses transnacionales diversos. La actuación, por ejemplo, de empresas transnacionales y diferentes lógicas de intervención directa del capital y del Estado en los territorios suelen ser foco de confrontación con luchas por la tierra y por el territorio, sean luchas de afectados por presas hidroeléctricas, empresas específicas o la minería transnacional, sean movimientos más articulados en dimensiones culturales.

Luchas vinculadas a derechos sociales - Todas aquellas luchas fundamentales para la profundización del proceso de democratización política y social de América Latina, vinculadas a la reivindicación de derechos básicos (educación, sanidad y vivienda, por ejemplo). Tienen un fuerte sentido de lo público y de apuesta democratizante frente a crecientes proyectos de conversión mercantil de los derechos. Tenemos, aquí, casos emblemáticos, como el de las luchas estudiantiles de Chile, pero también se pueden incluir muchas otras en toda la región. Si miramos las demás luchas de manera transversal, vemos también cómo emergen derechos de nuevo tipo, como, por ejemplo, el derecho a la alimentación y la soberanía alimentaria.
Luchas vinculadas a la reproducción de la vida, la memoria y la identidad - De manera paralela, y a veces convergente con el eje anterior, se incluye aquí un amplio abanico de movimientos culturales y de acciones desplegadas por verdad y justicia, vinculadas a organizaciones de derechos humanos y todo lo que significa la recuperación de la memoria y derechos de reconocimiento y de identidad, incluyendo la identidad sexual y todo lo relacionado a demandas de matrimonio homosexual, entre otros, como los derechos vinculados a la mujer en cuanto a interrupción voluntaria del embarazo.

La ausencia de un conflicto central en nuestras sociedades, en el siglo XXI, tal como enmarcado por los movimientos sociales durante buena parte del siglo XX, lleva a la dificultad de generación de un proyecto de sociedad con capacidad de agregación (Garretón, 2001). Principios de unificación son posibles, pero siempre considerando este marco general de diversidad de conflictos y actores, que transcurre de forma paralela a un cambio más amplio, de carácter sociocultural y generacional.

A partir de los ejes enunciados, aparecen algunos desafíos para los protagonistas de estas luchas sociales. Un primer reto consiste en generar inteligibilidad con actores no movilizados por los canales convencionales, a partir de temas que afectan dramáticamente a toda la sociedad, es decir, en generar visiones y canales societarios que trasciendan los propios grupos organizados, incluyendo la batalla cultural.

Otro desafío está relacionado a las posibilidades de complementariedad y de retroalimentación entre movimientos, partidos y gobiernos. Por ejemplo, en procesos sociopolíticos caracterizados como progresistas, los movimientos, en cada conflicto, deben procesar flujos permanentes y complejos de información, pero también deben tener la capacidad de neutralizar el poder del consenso de lo técnico sobre lo político. La llegada de gobiernos caracterizados como progresistas permitió, 
inicialmente, una mayor apertura de demandas y de agendas, pero también, y de forma contradictoria, en algunos casos, llevó a una mayor represión y falta de diálogo cuando se trataba de la implementación, por ejemplo, de megaproyectos (como carreteras, hidroeléctricas) donde, no pocas veces, los intereses de los gobiernos se vinculaban claramente a fines y compromisos electorales, como las subvenciones a campañas.

Asimismo, acrecentó una perspectiva de supuesta neutralidad técnica y pospolítica de la gestión que ha impregnado toda la sociedad. Para poder visualizar esto con mayor claridad, pasamos a abordar algunas de las contradicciones del progresismo, distinguiendo tipos de gobierno y su gestión de lo público en el contexto de cambios estructurales de la forma Estado antes aludida.

\section{TIPOS DE GOBIERNOS EN AMERI- CA LATINA: límites del progresismo, gestión de lo público y dinámicas de consenso o coerción}

Los estudios tradicionales de movimientos sociales tienden a defender la hipótesis, bastante intuitiva, de que la emergencia de gobiernos de centro-izquierda y de izquierda, con algún tipo de afinidad con los movimien$\rightarrow$ tos, abre un escenario de mayor apertura hacia ล sus agendas y demandas. El análisis de la diversidad de situaciones ocurridas en América ¿. Latina, desde el cambio de siglo, sin embargo, \% nos muestra un escenario bastante más com될 plejo, que apunta a dinámicas ambivalentes, a \& tensiones diversas, a límites del progresismo, oิ a modalidades distintas de gestión de lo públi$\vec{c}$ co, bien como a varias combinaciones posibles de apertura y cierre, consenso y coerción.

Más allá de los frenos geoeconómicos y de los tipos de conflictos sociales ya analizados, una interpretación multidimensional sobre esta temática tiene que contemplar también las formas de gestión, las relaciones históricas y subjetivas, las dinámicas político -ideológicas en el interior de las sociedades y algunas tendencias y eventos (geo)políticos. Pensemos, por ejemplo, en el golpe de Estado a Zelaya, en Honduras en 2010, y en las destituciones del presidente Lugo en Paraguay en 2012 y de la presidenta Rousseff en Brasil en 2016, acompañadas de un complejo entramado social en su potencialidad de respuesta. Cabe agregar, asimismo, una amplia lista de intentos fallidos de restauración conservadora en Ecuador (2010), Bolivia (2008) y Venezuela (2002) y avances de desestabilización política de signo conservador en varios otros países, que incluyen no sólo reacción desde arriba, sino también apelaciones a la movilización social en las calles.

Por otro lado, la ausencia de conflictos más marcada, en general, con el capital, en los casos de Brasil, Chile y Uruguay, evidencia procesos de contención del cambio potencial. La emergencia de un ciclo electoral con victorias de candidatos y (o) propuestas más conservadoras, que incluso pueden provenir de filas de los propios agentes políticos vinculados al gobierno, avala dicha tendencia. Todo esto sugiere que las alianzas o los acuerdos estratégicos, explícitos o no, con movimientos sociales pueden crujir fuertemente.

Delineados estos escenarios, corresponde traspasar el mero registro de acontecimiento y ensayar una tipología de casos considerando ese proceso socio-histórico que emerge con las luchas de la década de 1990 y que se complejiza en el cambio de siglo, con una diversidad de formatos sociopolíticos. El principal criterio utilizado será la gestión, en general, ya introducida en el apartado anterior, y la gestión del conflicto (real o potencial), en particular.

Antes de ello, una breve advertencia: toda propuesta de tipología genera inmediatamente preguntas y consideraciones sobre alternativas posibles a la misma. Siempre puede ser objetable el criterio central establecido o la razón por la cual un caso es incluido junto o separado de otro. La presente propuesta no 
busca una delimitación rígida, sino un marco general de observación que permita acotar nuestra discusión, marcando ciertas tendencias regionales asociadas a las limitaciones de acción de los gobiernos, principalmente en lo que se refiere a su gestión de lo público y a los cambios de la forma Estado. Considerando, entonces, las gestiones de gobierno - no sólo los progresistas - en la región en la actualidad, en los quince primeros años del siglo XXI, y la combinación de dinámicas de consenso y coerción, pueden diferenciarse cuatro formatos principales:

Gestión con autoritarismo selectivo y violencia extendida en el tejido social - México, Colombia y Centroamérica en general. En estos casos, los componentes de coerción han sido prominentes. También aquí pueden integrarse las intervenciones quirúrgicas como la de Honduras.

Gestión empresarial con gobiernos de derecha política - Chile con la coalición de centro derecha de Piñera, Paraguay con Cartes (del tradicional Partido Colorado pero con importante incorporación de técnicos conservadores) y Perú con Humala (o al menos el intento $\mathrm{y}$, en este caso, a partir de un giro que desdibuja una anterior perspectiva de cambio).

Gestión empresarial con gobiernos progresistas sin intento de transformación de la forma Estado heredada - Chile con la Concertación (y con la llamada Nueva Mayoría), Brasil con el PT, Uruguay con el Frente Amplio, Paraguay con la Alianza de Lugo.

Gestión contradictoria con intento de transformación de la forma Estado heredada - En esta situación, se observan ambivalencias y tensiones elásticas, con movimientos sociales como en el caso de Bolivia y Ecuador, bien como tensiones polares como en el caso de Argentina (peronismo) y Venezuela (chavismo). En este último caso, desde 2013, se ha entrado en un período de conflictividad y bloqueos importantes que actúan como freno a la potencialidad de otras transformaciones.

De dicha tipología, es importante tener en cuenta que hay casos en que se manifiesta claramente la capacidad de coerción en la gestión. Esto se traduce en la violencia de Estado y en la represión expuesta o invisibilizada, que puede ser esporádica, complementaria o sustitutiva del consenso (aunque esta última nunca puede ser por un período largo). La forma en que ésta despliega hoy la capacidad de coerción tiene más bien un carácter acotado y, de hecho, en sociedades marcadas por la violencia estructural (como Colombia), la tendencia es intentar pasar a otro esquema político.

En el siglo XX, las dictaduras que duraron décadas (Paraguay es un caso extremo) lograron hacer efectiva una mezcla de coerción y consenso, donde la primera tuvo un rol clave. En cambio, hoy, y tal como se señaló en la tipología, la coerción tiene más bien carácter quirúrgico, de extirpación rápida y localizada, incluso revestida jurídicamente, para volver a simular lo más rápidamente posible un orden consensuado, democrático, que permita la reproducción de determinados intereses económicos.

También conviene recordar, una vez más, que las situaciones en América Latina son muy diversas en las expresiones recientes de conflicto social. Se presentan, de este modo, oscilaciones que contemplan desde sociedades con tejido social activo, con capacidad de cuestionar líneas de gobierno (Bolivia y Ecuador), a veces con disputas fuertes entre agentes sociales con bases y perspectivas contrapuestas entre reaccionarias y transformadoras (Argentina y, sobretodo, Venezuela), con mezcla de rebeliones puntuales y movimientos sociales más estructurados (Brasil, Chile y México), o casos como el de Uruguay, en el que aparece un tejido social debilitado y contenido (aspecto que resalta también en función de su escala en comparación con sus vecinos) y con incapacidad de proyectar otros horizontes de posibilidades sobre lo dado ${ }^{3}$. ${ }^{3}$ Cabe, sin embargo, matizar el caso a partir del 2015, en
que la lucha por el presupuesto - principalmente en edu-
cación - ha implicado movilizaciones por demandas muy
importantes, inéditas durante el período de gobierno del
Frente Amplio, que abren numerosas preguntas. 
En definitiva, si entendemos que los movimientos sociales, en América Latina, disputan, en sus diversos planos de relación con el Estado y con la sociedad, tanto proyectos políticos como proyectos de sociedad, es fundamental tratar de captar los movimientos societarios más amplios, las limitaciones de la potencialidad transformadora de los gobiernos, la reconfiguración de lo público y sus interacciones.

Hasta aquí se ha tratado de llamar la atención para las transformaciones contemporáneas de la forma Estado en América Latina y las perspectivas de gestión gubernamental de lo público, con sus respectivas implicaciones para los movimientos sociales y para la sociedad. No obstante, en la teoría y en la práctica, el Estado debe considerarse siempre como un actor dual: en un extremo, sigue teniendo el monopolio legítimo de la violencia en una territorialidad delimitada, donde ejerce su poder, la coerción, e influencia sobre actores diversos; en el otro, es también el responsable por la democratización política y social, jugando un papel distinto de interlocución con la sociedad y los movimientos (visto, en general, como normativamente más positivo). En el medio de estos dos polos, una multitud de formatos de interacción son posibles.

\section{CONEXIONES, (RE)CONFIGURA- CIONES Y MEDIACIONES: gobier- nos, partidos y movimientos}

entre Estado y sociedad

今 con bastante peso del primero, que jugó un pa-

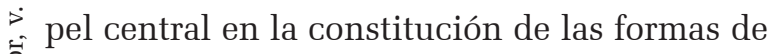
movilización, relación y surgimiento de muchos actores sociales. La matriz nacional-popular clásica, que se desarrolla en su formato tradicional hasta la década de 1960, expresa, de manera clara, un ejemplo de fuerte imbricación y co-implicación entre sus componentes
(Estado, gobiernos, partidos y actores sociales) en el marco de una forma específica de hacer política y construir mediaciones y vínculos institucionales. ${ }^{4}$

La configuración de esta matriz sociopolítica se sostuvo en una tradición político-ideológica que sostenía la centralidad de un Estado redistributivo, conciliador, con una participación política controlada y líderes carismáticos. Obviamente, no se trataba de una lógica unívoca de mediación, ya que también hay que entender la constitución de otras narrativas paralelas y en disputa, como la indígena-comunitaria. De todos modos, lo que nos interesa subrayar es que esta matriz sociopolítica se asociaba también a una inserción o configuración económica y a un tipo societario predominante en América Latina, presente durante buena parte del siglo $\mathrm{XX}$, que entró en crisis.

La llegada de las dictaduras y la posterior emergencia del Estado transnacionalizado implicó también una rearticulación de formas distintas de relación entre Estado, actores de mediación y movimientos sociales. Tenemos, así, una mayor diversidad de actores sociales, un énfasis más fuerte en la democratización social, una reformulación del modelo de desarrollo, una mayor interpenetración de proyectos y modelos societarios, bien como una creciente transversalización de las lógicas de acción colectiva y de contestación. Las percepciones sobre el Estado y las concepciones de cambio social también se han transformado profundamente en la región, en un momento de transición, donde, en algunos lugares, han emergido sujetos políticos que buscaron generar nuevos instrumentos políticos de mediación entre Estado y sociedad (casos de Bolivia y Ecuador con el Movimiento al Socialismo (MAS) y con el Movimiento de Unidad Plurinacional Pachakutik (MUPP), pero también en el caso fallido del Partido Popular Tekojoja (PPT) en

${ }^{4}$ Los trabajos de Fernando Calderón, Elizabeth Jelin y Manuel Antonio Garretón son emblemáticos para entender esta relación desde el punto de vista de los actores sociales, mientras los trabajos de Juan Carlos Portantiero, en Argentina, y Francisco Weffort, en Brasil, lo son para una óptica más centrada en los Estados. 
Paraguay). En otros casos, hay una enorme dificultad de convivencia y mediación de actores que surgen en los contextos de lucha contra la dictadura, como instrumentos centrales de democratización, pero se ven incapaces, en el gobierno, de generar vínculos más sólidos con las bases sociales (casos de Brasil con el PT, Uruguay con el Frente Amplio, por quedarse sólo en dos ejemplos muy conocidos).

Dentro de toda esta complejidad y siguiendo con la idea de abrir planos teóricometodológicos posibles, si se examinan las diferentes mediaciones que se han establecido en los últimos años en América Latina entre gobiernos, partidos y movimientos, puede proponerse una nueva mirada transversal que busca observar variaciones dentro de un abanico de situaciones posibles. Éstas pueden desplazarse más rápidamente entre uno y otro casillero, dependiendo de la coyuntura. La propuesta abajo sirve como una guía abierta para complejizar situaciones que van más allá de las habituales dicotomías social-democracia y populismo, cuando se hace referencia a los gobiernos progresistas en la región (algo reforzado por la tesis reciente de las dos izquierdas) o entre cooptación o autonomía, en lo que atañe a las relaciones entre movimientos sociales y gobiernos (visión habitual dentro de análisis normativos sobre el lugar y el papel de los actores sociopolíticos):

Conexión subordinada - Casos en los que se establecen relaciones fuertes entre gobierno, partido y movimientos, donde la separación de actuación entre los espacios sociales no es clara, y se puede obtener demandas por los movimientos, pero a costo de estar sujetos a lógicas muchas veces vinculadas a una reproducción neocorporativa. En este caso, no solo el Gobierno o el partido tienen fuerte influencia en las decisiones de los movimientos, sino que prescriben el marco de acción en el que se mueven. Aquí pueden entrar situaciones tan diferentes como la Confederación General del Trabajo (CGT) durante Menem en Argentina, o Venezuela en la relación con el gobierno y
Partido Socialista Unido de Venezuela (PSUV).

Conexión negociada - Situaciones en las que el extremo anterior no se alcanza, pues se observa separación entre los tres planos. Se observa un control puntual sobre miembros de los movimientos, aunque no del agente colectivo como un todo. En este caso, se tiene un canal de acceso privilegiado al gobierno, pero el costo sigue siendo alto en lo que se refiriere a la autonomía, dado que el campo político es bastante semejante y las relaciones históricas muy fuertes. Puede ser el caso de movimientos sindicales en el contexto de gobiernos progresistas del cono sur, como la Central Única de los Trabajadores (CUT) en Brasil, entre otros.

Conexión discontinuada - Escenarios en los que el caso anterior se alterna con situaciones diversas, y en donde se presentan y se reemplazan contextos de compromiso del movimiento con contextos de luchas y movilizaciones. Son los típicos casos en que hay un horizonte (práctico y discursivo) de autonomía, pero, por diferentes causas, incluyendo lazos personales, afinidades y trayectorias comunes, no se renuncia a la conexión con el gobierno. La relación con los partidos existe pero es más débil. Incluye casos como los de Federación Uruguaya de Cooperativas de Vivienda por Ayuda Mutua (FUCVAM) en Uruguay, Movimiento de los Sin Tierra (MST) en Brasil, movimientos indígenas y campesinos en la región andina. Es el caso más volátil de los cuatro.

Desconexión con gobiernos y presencia débil o inexistente de partidos - Situación en que la autonomía del movimiento es más clara. Y puede serlo por un contexto claramente adverso del gobierno (federal, estadual o ambos), como el Ejército Zapatista de Liberación Nacional (EZLN) en México, o porque las posturas del propio movimiento, en cuanto a visión general de la realidad, tienden a hacer incompatible cualquier conexión con gobiernos o partidos. Es el caso de algunos movimientos de base indígena, cuya cosmovisión aparece como claramente diferenciada de cualquier lógica neodesarrollista, más allá de 
su sensibilidad social o incluso de narrativas emancipatorias que puedan acercarles a gobiernos y (o) partidos. En estos casos, la autonomía es un elemento subjetivo fuerte y se ejercita en la vivencia personal y en la praxis colectiva, pero lleva a que no se pueda apelar a negociaciones como recurso estratégico para las demandas del movimiento.

El presente esquema puede contribuir a entender relaciones que, a primera vista, son profundamente ambivalentes. Por ejemplo: ¿Cómo explicar que movimientos que son afectados en forma significativa por las políticas de gobiernos supuestamente progresistas sigan defendiendo estos mismos gobiernos? Esto puede ocurrir en los casos de conexión descontinuada, por ejemplo, cuando buena parte de los movimientos campesinos en Brasil, a pesar de la priorización dada por el gobierno del PT al agronegocio, seguían apoyándolo. A pesar de un escenario objetivamente negativo (represión, apertura restringida a sus demandas, deterioro de las condiciones de la vida en el campo, avance del monocultivo de exportación), las relaciones históricas y subjetivas, la pertenencia a un campo común de acción o el aumento de la polarización política (que lleva a la adopción del discurso de lo menos peor, bajo el riesgo de mayores retrocesos) pueden ser elementos justificadores de las relaciones ambivalentes en este y en otros casos.

De este modo, como consecuencia de la tipología propuesta, sugerimos que, en la explicación de estas situaciones complejas, habría que considerar, al menos, cuatro elementos fundamentales: a) los vínculos objetivos (acciones concretas, políticas públicas y datos o elementos que permitan vislumbrar, e incluso mensurar, las relaciones mediadas por agendas, discursos y políticas); b) las relaciones subjetivas (convergencias y afinidades históricas, ideológicas, personales, biográficas y de trayectorias que llevan a la elaboración de determinados marcos cognitivos, sentimentales y morales); c) los movimientos societarios (transformaciones coyunturales y estructu- rales de la sociedad y visiones de la población no necesariamente organizada en movimientos, pero que influyen en las conductas y prácticas sociales, bien como en actitudes políticas transversales, como puede ser el caso de las polarizaciones acentuadas); d) el escenario geo-político y geoeconómico, que marca patrones específicos de inserción en la economía capitalista y en las dinámicas regionales y globales.

\section{REFLEXIONES FINALES}

Tras casi dos décadas de relaciones sinérgicas entre izquierda política y social, América Latina vive un momento delicado: muchos de los gobiernos de izquierda que se eligieron en el cambio de siglo, con el apoyo de los movimientos sociales, no sólo no consumaron sus demandas más rupturistas, sino que, en muchos casos, ahora también los reprimen. Otros empiezan a perder elecciones o son destituidos formalmente con diversos formatos de desestabilización y de golpes jurídico-mediáticos.

Prioridad para la mayoría de los gobiernos, las políticas sociales estuvieron en el centro del debate sobre los Estados (refundados o no), confluyendo, en general, con un continuismo, en el terreno económico, con los dictámenes del capitalismo global que refuerzan la posición periférica de América Latina en el sistema mundial.

Las posibilidades de conexiones examinadas entre movimientos y gobiernos (subordinada, negociada o discontinuada), o incluso la desconexión, se relacionan con vínculos objetivos y relaciones subjetivas, bien como con tipos de gestiones en las que priman una lógica empresarial y combinaciones de dinámicas de coerción y de consenso. Como consecuencia, se delinean conflictos y luchas sociales específicas, que deben ser comprendidas en una lógica procesual y relacional. Las transformaciones en curso actualmente en la región, y que permiten proyectar nuevos conflictos y tensiones, sugieren también nuevas distribuciones 
de casos dentro de la tipología de conexiones esbozada.

En cuanto a la problemática de captación, al subrayar estos elementos y generar tipologías transversales, se ha buscado, en este artículo, romper con algunas tendencias habituales y problemáticas en el estudio de los movimientos sociales y su relación con el Estado: a) el análisis meramente coyuntural y de corto plazo, que no tiene en cuenta coordinadas temporales y estructurales más amplias; b) el empirismo casuístico que, si bien enriquece el conocimiento sobre movimientos sociales y dinámicas micro, no es capaz de generar un marco analítico más amplio, necesario para entender los sujetos en su totalidad societaria; c) el cierre de los movimientos sociales en sí mismos, que busca analizar los movimientos sociales como un fin, siendo incapaz de abordar un campo relacional más amplio, las tensiones entre diferentes movimientos sociales y entre éstos y otros actores políticos y económicos que disputan proyectos políticos y societarios; d) la construcción de categorías analíticas basadas en las experiencias del Norte, que universalizan formas de entender los movimientos sociales y el Estado a partir de matrices particulares (europeas o norte-americanas) y teorías eurocéntricas.

Un desafío teórico-metodológico clave es la necesidad de abordar la transformación de la forma Estado a partir del accionar de los movimientos sociales sobre los diferentes tipos de gobiernos y en función de otras dinámicas regionales y globales. Al transitar entre diversos escenarios y manejar varias variables, algunas preguntas se fortalecen como campos de investigación privilegiados en el futuro próximo: ¿Cómo incide el tipo de gobierno en el patrón de relaciones con los movimientos sociales? ¿Cómo asociar ciclos político-electorales con ciclos de movilización?

Estas y otras preguntas aquí planteadas nos llevaron a transitar por un terreno de investigación lleno de obstáculos, que solo se hacen visibles al transitarlo. Si, en la década de 1960 y principios de 1970, la región generó aportes claves a partir de imaginativas aperturas de nuevos planos de análisis, no resulta descabellado pensar que hoy bien podríamos reencontrarnos con ese legado teórico-metodológico para construir otras bases que permitan explicar las nuevas realidades. En América Latina, existen contextos muy diferentes de producción de conocimiento en las Ciencias Sociales y podrían caber aquí generalizaciones abusivas. Pero una cosa es segura: si la lógica pragmática, ultra-especializada e instrumental de construcción de conocimiento ahoga la reflexión y la capacidad de abrirse a mediaciones analíticas, será cada vez más difícil concretar la invitación de este texto y forjar interpretaciones multidimensionales y abarcadoras de la región. Más estudios comparativos y transversales, empíricamente orientados pero con pretensión teórica, serán muy bienvenidos para seguir avanzando en esta agenda de investigación.

Recebido para publicação em 15 de outubro de 2015 Aceito em 21 de novembro de 2015

\section{REFERENCIAS}

ABERS, R.; SERAFIM, L.; TATAGIBA, L. "Repertórios de interação Estado-sociedade em um Estado heterogéneo: a experiência na Era Lula”, Dados - Revista de Ciếncias Sociais. 2014, v. 57, n. 2, p. 325-357.

AMIN, S. Más allá del capitalismo senil. Por un siglo XXI no norteamericano. Buenos Aires: Paidós. 2003.

BOLTANSKI, L.; CHIAPELLO, E. El nuevo espíritu del capitalismo. Madrid: Akal (1 ${ }^{\mathrm{a}}$ edición en francés 1999). 2002.

BORBA, P. Sociologia histórica como teoria política: a formação dos Estados modernos na Europa e na América Latina. Dissertação de Mestrado (Programa de Pós-Graduação em Ciência Política). Rio de Janeiro: IESP-UERJ. 2014.

BOURDIEU, P.; WACQUANT, L. "Una invitación a la Sociología reflexiva", Buenos Aires, Siglo Veintiuno editores. 2014 [1992].

BRINGEL, B. Social movements and contemporary modernity: internationalism and patterns of global contestation. In: BRINGEL, B.; DOMINGUES, J. M. (Orgs.) Global modernity and social contestation. Londres/ California/New Delhi: SAGE, 2015, p.122-138.

; CABEZAS, A. Geopolítica de los movimientos sociales latinoamericanos: espacialidades, ciclos de contestación y horizonte de posibilidades. In: PRECIADO, 
J. (Ed.) Anuario de la Integración Latinoamericana y Caribeña. Guadalajara/New Orleans: Ediciones de la Noche/University Press of the South, 2014, p. 323-342.

; DOMINGUES, J. M. Teoria crítica e movimentos sociais: intersecões, impasses e alternativas. In: BRINGEL, B.; GOHN, M. G. (Orgs). Movimentos Sociais na Era Global. Rio de Janeiro / Petrópolis: Vozes, 2012, p. 47-76.

(Eds.) Global modernity and social contestation. Londres/California/Nueva Delhi: Sage. 2015.

; FALERO, A. Redes transnacionais de movimentos sociais na América Latina e o desafio de uma nova construção socioterritorial. Caderno $C R H, 2008$, n. 53, p. 269-288.

CAIRO, H. A América Latina nos modelos geopolíticos modernos: da marginalização à preocupação com sua autonomía. Caderno CRH, 2008, v. 21, n. 53, p. 221-237.

CAMPELLO, D. The politics of market discipline in Latin America. Cambridge: Cambridge University Press. 2016.

CASTAÑEDA, J. Latin America's Left Turn. Foreign Affairs, 2006, v. 85 , n. 3, p. 28-43.

CENTENO, M. Blood and debt: war and the Nation-State in Latin America. Pennsylvania (University Park): The Pennsylvania State University Press. 2002.

DELLA PORTA, D. (Ed.) The Global Justice Movement: cross-national and transnational perspectives. Boulder: Paradigm Publishers. 2007.

FALERO, A. Problemas contemporáneos en la proyección de la teoría social y algunos desafíos inmediatos, ponencia presentada en el Primer Congreso Latinoamericano de Teoría Social, Buenos Aires, Instituto de Investigación Gino Germana, Facultad de Ciencias Sociales, Universidad de Buenos Aires, 19 al 21 de agosto. 2015.

Entre o rigor teórico-metodológico e a criatividade - Algumas chaves cognitivas para a pesquisa dos movimentos sociais na América Latina. In: BRINGEL, B.; GOHN, M. G. (Orgs). Movimentos sociais na Era Global. Rio de Janeiro/Petrópolis: Vozes, 2012a.

Una creativa travesía por el sistema-mundo. Las aperturas cognitivas de Arrighi para el estudio de procesos globales. In: LIMA, M. C.; VILLA, R.; MEDEIROS, M. A.; REIS, R. R. (Orgs.) Teóricos das Relações Internacionais. São Paulo: Hucitec, 2012b, p. 254-285.

Capitalismo y enclaves: nuevas dinámicas, viejos problemas, renovados desafíos para pensar alternativas. In: VVAA. Pensar a Contracorriente. La Habana: Editorial $\stackrel{\bullet}{*}$ de Ciencias Sociales, 2012c, p. 265-293.

FLORES, A. "Empresarios e izquierda: dos mundos que N se acercan", Revista Nueva Sociedad (Caracas), n. 202, is Editorial Nueva Sociedad, 2006, p. 157-168.

งิ FOUCAULT, M. [1976] Defender la Sociedad. México D.F.: ¿. Fondo de Cultura Económica. 2010.

§ GARRETÓN, M. A. Cambios sociales, actores y acción colectiva en América Latina. Serie Políticas Sociales के CEPAL, n. 56, Santiago de Chile. 2001.

¿ GRAMSCI, A. La política y el Estado moderno. Barcelona, of Planeta-Agostini. 1985 [1949].

$>$ LAVALLE, A. G.; BULLOW, M. V. Institutionalized Brokers if and Collective Actors: Different Types, Similar Challenges.
In: ROSSI, F.; BÜLOW, M. V. (Eds.). Social Movement Dynamics: New Perspectives on Theory Research from Latin America. Farnham: Ashgate, 2015, p. 180-225.

LINERA, A. G. La potencia plebeya. Acción colectiva e identidades indígenas, obreras y populares en Bolivia. Buenos Aires: CLACSO/Prometeo. 2008.

LÓPEZ-ALVES, F. La formación del Estado y la democracia en América Latina. Bogotá: Editorial Norma. 2003.

LOSEKANN, C. Ambientalistas em Movimento no Brasil: entrelaçamentos e tensões entre o estado e a sociedade durante o governo Lula. Curitiba: Editora Appris. 2014.

MANN, M. The Autonomous power of the State: its nature, causes and consequences. European Journal of Sociology, 1984, 25, n. 2, p. 185-213

McADAM, D.; TARROW, S.; TILLY, C. Dynamics of contention. Cambridge: Cambridge University Press. 2001.

MIRZA, C. A. Movimientos sociales y sistemas políticos en América Latina: la construcción de nuevas democracias. Buenos Aires: CLACSO. 2006.

NASCIMENTO, E. C. Mudanças e continuidades no movimento de direitos humanos: padrões organizacionais, relacionais e discursivos. Opinião Pública, Campinas, 2014, v. 20, n. 3, p. 450-479.

O’DONNELL, G. El Estado burocrático autoritario: triunfos, derrotas y crisis. Buenos Aires: Editorial de Belgrano. 1982.

SILVA, M. K. Atores, Espaços e Repertórios: a atuação dos movimentos sociais através das fronteiras da sociedade civil e do Estado. In: SCHERER-WARREN, I.; LÜCHMANN, L. H. H. (Orgs.) Movimentos Sociais e Engajamento Político: trajetórias e tendências analíticas. Florianópolis: Editora UFSC, 2015, p.133-160.

SILVA, P. da. Vitórias na crise: trajetórias das esquerdas latino-americanas. Rio de Janeiro: Ponteio. 2011.

PLEYERS, G. Internacionalização sem institucionalização? In: BRINGEL, B.; GOHN, M. G. (Orgs). Movimentos Sociais na Era Global. Rio de Janeiro / Petrópolis: Vozes. 2012.

POULANTZAS, N. Poder político y clases sociales en el Estado capitalista. México, Siglo XXI. 1986 [1968].

SANTOS, B. S. Refundación del Estado en América Latina. Lima: Instituto Internacional Derecho y Sociedad / Programa Democracia y Transformación Global. 2010, c. 6.

SCHERER-WARREN, I. Redes e movimentos sociais projetando o futuro. Revista Brasileira de Sociologia, 2013, v. 1, n. 1, p. 187-217.

SVAMPA, M. Consenso de los commodities y lenguajes de valoración en América Latina. Revista Nueva Sociedad (Caracas), n. 244, Editorial Nueva Sociedad, 2013, p. 3046

Movimientos sociales, matrices socio-políticas y nuevos escenarios en América Latina. Working Papers 01/2010, Kassel, Universitat Kassel. 2010.

TARROW, S. The new transnational activism. Cambridge: Cambridge University Press. 2005.

VON BULOW, M. Building transnational networks. Civil society and the politics of trade in the Americas. Cambridge: Cambridge University Press. 2010. 


\section{SOCIAL MOVEMENTS, PROGRESSIVE GOVERNMENTS AND STATES IN LATIN AMERICA: transitions, conflicts and mediations}

\author{
Breno Bringel \\ Alfredo Falero
}

This article analyzes contemporary Latin America from a procedural and relational perspective, from the interactions between social movements, progressive governments and the State. The goal is to understand the political cycle that begun in this century in that area vis-à-vis greater changes in the political, social and economic scope. More specifically, the text discusses the need to capture the social process and the diversity of types of interaction between social movements, governments and State; the specificity of the area, its dependent position in the world and social and political consequences this brings, including a transformation of the State model, which is increasingly trans-nationalized; the main axes of social conflict, originated mainly from social-political mediations and politicaleconomic contradictions; and, finally, the types of government, the limitations of progressivism and the tensions between state management and social movements.

KeYwORDS: Social movements. Progressive governments. State. Latin America.

\section{LES MOUVEMENTS SOCIAUX, LES GOUVERNEMENTS PROGRESSISTES ET LES ÉTATS EN AMÉRIQUE LATINE: transitions, conflits et médiations}

\author{
Breno Bringel \\ Alfredo Falero
}

Cet article se veut d'analyser l'Amérique Latine contemporaine en tant que clé d'un processus et de relations nées des interactions entre les mouvements sociaux, les gouvernements progressistes et l' État. L'objectif est de comprendre le cycle politique qui a commencé dans la région à l'aube de ce nouveau siècle face à des changements plus amples dans les domaines politique, social et économique. Ce texte traite plus spécifiquement: 1) du besoin de saisir le processus social et la diversité des formats d'interaction entre les mouvements sociaux, les gouvernement et l'Etat; 2) de la spécificité de la région, de sa position de dépendance par rapport au monde et aux conséquences sociales et politiques y relatives, y compris la transformation du modèle d'Etat, de plus en plus trans-nationalisé; 3) des principaux axes de conflits sociaux survenant essentiellement des médiations socio-politiques et des contradiction politiques et économiques, et enfin 4) des types de gouvernement, des limites du progressisme et des tensions entre la gestion de l'État et les mouvement sociaux.

Mot-CLÉs: Mouvements sociaux. Gouvernements progressistes. État. Amérique Latine.

\begin{tabular}{l} 
Breno Bringel - Doutor em Ciência Política. Professor do Instituto de Estudos Sociais e Políticos da \\
Universidade do Estado do Rio de Janeiro (IESP-UERJ) e Directeur d'études associé no Collège d'études \\
mondiales da Fondation Maison des Sciences de l'homme em Paris. Bolsista de Produtividade em \\
Pesquisa do CNPq. Editor de Dados-Revista de Ciências Sociais e de OpenMovements. Coordenador \\
do Núcleo de Estudos de Teoria Social e América Latina (NETSAL). Temas de interesse: movimentos \\
sociais; internacionalismo; teoria social; América Latina. Publicações recentes: Global modernity and \\
social contestation (com José Maurício Domingues, Londres/New Delhi, Sage, 2015); Teoria social, \\
extroversão e autonomia: impasses e horizontes da sociologia (semi)periférica contemporânea. Caderno \\
CRH (Online), v. 28, p. 59-76, 2015. \\
Alfredo Falero - Doutor em Ciências Sociais. Professor/pesquisador da Universidad de la República \\
(UDELAR) no Uruguai. Foi professor visitante do IESP-UERJ em 2014. Coordenador do Núcleo \\
Interdisciplinar de Pensamento Crítico (2013-2015) e do projeto “Territórios Controlados" (2015-2016). \\
Ganhou o concurso "Los legados teóricos de las Ciencias Sociales en América Latina y el Caribe” do \\
CLACSO, na categoria pesquisador sênior. Temas de interesse: processos globais; América Latina; \\
movimentos sociais; pensamento e teoria social. Editor de Movimientos sociales, educación popular \\
y trabajo autogestionado en el Cono Sur (2013), entre outros. Autor dos livros Las batallas por la \\
subjetividad (2008); Los enclaves informacionales de la periferia capitalista (2011). \\
\hline
\end{tabular} 
